\title{
Erratum to: Media and Technology \\ Use Among Hispanics/Latinos in New York: Implications for Health Communication Programs
}

\author{
Jennifer A. Manganello ${ }^{1}$ - Gena Gerstner ${ }^{2}$ - Kristen Pergolino ${ }^{2}$ - Yvonne Graham ${ }^{2}$. \\ David Strogatz ${ }^{3,4}$
}

Published online: 31 December 2015

(C) W. Montague Cobb-NMA Health Institute 2016

Erratum to: J. Racial and Ethnic Health Disparities

DOI 10.1007/s40615-015-0169-9

The following acknowledgements were inadvertently omitted:

The authors acknowledge Siena Research Institute for data collection as well as Jessica Coyle and Angela Falisi for their contributions as Graduate Student Interns from the University at Albany, School of Public Health. Student efforts were supported by the Empire State Public Health Training Center within the Center for Public Health Continuing Education at the University at Albany, School of Public Health.

The online version of the original article can be found at http://dx.doi.org/ 10.1007/s40615-015-0169-9.

Jennifer A. Manganello

jmanganello@albany.edu

1 Department of Health Policy, Management, and Behavior, University at Albany School of Public Health, Rensselaer, NY, USA

2 Office of Minority Health and Health Disparities Prevention, New York State Department of Health, Albany, NY, USA

3 Department of Epidemiology and Biostatistics, University at Albany School of Public Health, Rensselaer, NY, USA

4 Center for Rural Community Health, Bassett Research Institute, Cooperstown, NY, USA 